Questions vives

\section{Questions Vives}

Recherches en éducation

$n^{\circ} 22 \mid 2014$

Questionner le curriculum en éducation physique et sportive : Quelle dynamique en contexte? Quelle autonomie?

\title{
Le rapport aux œuvres en danse au collège : contrainte et autonomie du curriculum. Le cas de la monstration de la proposition dansée
}

Relationships to dance artwork in middle school: constraints and autonomy of the curriculum. The case of teaching of a dance movement

Dominique Montaud et Chantal Amade-Escot

\section{(2) OpenEdition \\ 12 Journals}

\section{Édition électronique}

URL : http://journals.openedition.org/questionsvives/1628

DOI : $10.4000 /$ questionsvives. 1628

ISSN : $1775-433 \mathrm{X}$

Éditeur

Université Aix-Marseille (AMU)

Édition imprimée

Date de publication : 15 décembre 2014

ISBN : 978-2-912643-46-9

ISSN : 1635-4079

\section{Référence électronique}

Dominique Montaud et Chantal Amade-Escot, «Le rapport aux œuvres en danse au collège :

contrainte et autonomie du curriculum. Le cas de la monstration de la proposition dansée », Questions Vives [En ligne], $n^{\circ} 22$ | 2014, mis en ligne le 16 février 2015, consulté le 19 avril 2019. URL : http:// journals.openedition.org/questionsvives/1628; DOI : 10.4000/questionsvives.1628

Ce document a été généré automatiquement le 19 avril 2019.

\section{cc) (†)}

Questions Vives est mis à disposition selon les termes de la licence Creative Commons Attribution -

Pas d'Utilisation Commerciale - Pas de Modification 4.0 International. 


\title{
Le rapport aux œuvres en danse au collège : contrainte et autonomie du curriculum. Le cas de la monstration de la proposition dansée
}

\author{
Relationships to dance artwork in middle school: constraints and autonomy of \\ the curriculum. The case of teaching of a dance movement
}

Dominique Montaud et Chantal Amade-Escot

\section{Contexte et problématique de la recherche}

S'appuyant sur une recherche longitudinale portant sur la manière dont un enseignant d'éducation physique et sportive (EPS) enseigne la danse dans le cadre de l'enseignement obligatoire du collège (Montaud, 2014), cet article examine la manière dont les préconisations curriculaires en EPS relatives à « la formation artistique des élèves » trouvent place dans un enseignement de la danse au collège (MEN, 2008a). En effet, les discours officiels ainsi que les documents d'accompagnement des programmes mettent en avant les dimensions artistiques, corporelles et de communication de la danse contemporaine au regard des rôles de danseur, de chorégraphe et de spectateur. La primauté de l'intention, la nécessité d'une production chorégraphique singulière, la recherche de la divergence grâce au processus de symbolisation et l'accès au patrimoine culturel sont au cœur des compétences attendues chez les élèves. Il s'agit aussi de former le futur citoyen cultivé en contribuant au développement de sa culture artistique, en favorisant l'acquisition chez l'élève de valeurs humanistes, de tolérance, de respect de l'altérité et de la différence. L'EPS contribue ainsi à l'acquisition d'une culture humaniste, cinquième pilier du socle commun des programmes du collège. Comment ces intentions curriculaires ambitieuses sont-elles mises en œuvre? 
2 Cette recherche se centre sur l'évolution des savoirs mis à l'étude par un enseignant d'EPS sur une durée de six ans, lors de quatre cycles d'enseignement de la danse avec des classes de sixième (11-12ans). Elle débute à un moment particulier de l'évolution du curriculum d'EPS en France, celui où l'accent est mis sur l'importance de la construction d'un « rapport culturel aux œuvres ». L'introduction en 2008 de nouveaux programmes au collège en EPS et en Histoire des arts (MEN, 2008a, 2008b) a ainsi constitué un contexte favorable à l'investigation du «curriculum en acte(s) » thème central de ce numéro de Questions Vives. Cette étude de cas s'attache à identifier l'évolution des savoirs mis à l'étude par un même enseignant sur plusieurs années, afin de rendre compte des modalités de mise en œuvre des injonctions curriculaires.

\subsection{Terrain de l'étude}

3 Sur les six années de la recherche, nous avons observé un enseignant (que nous appellerons Pierre) ayant sept ans d'expérience d'enseignement en EPS mais aucune dans celui de la danse et qui, pour mettre en œuvre les nouveaux programmes, a suivi pendant cette période trois stages de formation continue en danse contemporaine.

4 Deux tâches prototypiques d'une leçon de danse (au sens de Harbonnier-Topin, 2012) ont été systématiquement analysées lors de la recherche: l'enseignementapprentissage d'une «proposition dansée» élaborée par l'enseignant; la "performation d'une chorégraphie » composée par les élèves et sa monstration devant leurs pairs. Ces deux moments, mobilisent des dimensions curriculaires relatives à l'instauration de nouveaux rapports aux œuvres en danse. Dans le cadre de cet article nécessairement restreint, nous nous intéressons plus particulièrement à l'évolution des savoirs mis à l'étude lors de l'enseignement apprentissage de la proposition dansée, tâche récurrente mise en place dans toutes les séances observées pendant les six années. Selon Harbonnier-Topin (2012), cette tâche, prototypique de l'enseignement de la danse dans les conservatoires comme au collège, a pour fonction de permettre aux élèves d'acquérir un vocabulaire gestuel, de construire des éléments de profondeur constitutifs du mouvement dansé, susceptibles d'être réinvestis lors de la composition chorégraphique qu'ils devront performer devant leurs pairs. L'analyse des savoirs réellement mis à l'étude au fil des ans et donc de leur évolution a mis en évidence les contraintes et l'autonomie du processus d'enseignement-apprentissage au regard des injonctions du programme d'EPS. Quelles sont-elles?

\subsection{La danse en EPS : une définition mal assurée}

5 L'institution scolaire assigne à l'EPS de penser les pratiques scolaires de la danse en référence au champ des pratiques artistiques contemporaines de ce domaine. Mais qu'est-ce que la danse? Comment la définir? Sur quels fondements assurer sa transposition? Les programmes d'EPS (MEN, 2008a) proposent une approche contradictoire en mettant en avant ses visées de formation artistique et d'accès aux œuvres culturelles, tout en la classant dans le cadre de la "compétence propre 3 » (« réaliser une prestation corporelle à visée artistique ou acrobatique ») réunissant des activités physiques, sportives et artistiques aussi différentes que la gymnastique sportive, la gymnastique rythmique, les arts du cirque, l'aérobic, etc. (MEN, 2008a). La définition d'usage, dans les traités didactiques et dans les textes officiels, est de considérer la danse comme une «activité physique d'expression, 
d'évocation du réel, destinée à être vue et à produire de l'émotion, de la surprise, à communiquer des sentiments grâce à la production de formes corporelles " (MEN 1996). Cette définition n'indique pas clairement sous quelles conditions un geste prend le statut de geste dansé, ni comment un œil expert en danse peut déclarer face à deux productions d'élèves qui, sur le plan de la forme, sont à peu près identiques : «là ça bouge; là ça danse ». De plus, nous considérons que le manque de précision de cette définition ne permet pas de différencier les pratiques dites artistiques, comme la gymnastique ou la natation synchronisée ${ }^{1}$, de la danse; ni de spécifier les propriétés du geste dansé. Elle n'éclaire pas non plus la question de la confusion entre danse et expression corporelle souvent de mise en milieu scolaire (Arguel, 1992). Plus largement, elle ne distingue pas la danse comme ensemble de pratiques et d'expériences personnelles de l'art chorégraphique ni ne précise ce qu'est l'art de danser. Ces questions de définition taraudent pourtant les didactiques des disciplines artistiques (Mili \& Rickenmann, 2004, 2005) car elles sont centrales pour analyser, comprendre, penser les modalités de l'enseignement artistique en milieu scolaire. C'est pourquoi, face à cette définition mal assurée du curriculum officiel, il est nécessaire de croiser une réflexion épistémologique et philosophique à propos des œuvres et du rapport aux œuvres, pour, dans un deuxième temps mener une analyse ascendante de la transposition didactique (Schubauer-Leoni \& Leutenegger, 2005) de la danse au collège afin de rendre compte des contraintes et de l'autonomie du curriculum tel qu'acté par le professeur et les élèves. Dans ce cadre, nous mobilisons les descripteurs de l'action conjointe en didactique, présentés dans la section suivante, pour rendre compte de ce processus performatif lors de la co-construction des contenus d'enseignement par Pierre et ses élèves de sixième dans un moment particulier, celui de la transmission d'une proposition dansée.

\section{Cadre théorique et méthodes}

6 Pour rendre compte de l'évolution des savoirs mis à l'étude par Pierre dans cet enseignement, nous nous sommes attaché à décrire sa pratique à un grain d'analyse très fin, de type micro-didactique afin de saisir les modalités singulières d'émergence du curriculum effectif (Crahay, Audigier \& Dolz, 2006; Harlé, 2009). Il s'agissait de débusquer - au-delà des formes récurrentes de "monstration-reproduction » (Harbonnier-Topin, 2012) qui selon cette auteure caractérisent ce moment particulier des séances de danse - la manière dont sont co-construits, en classe, les rapports aux œuvres en danse. Focaliser sur la dimension artistique et culturelle préconisée par les textes suppose en amont de clarifier ce qu'est une « œuvre » en danse. Selon les canons de la recherche didactique, cette étape relève d'une analyse épistémologique de l'objet d'enseignement, ici fondée sur la littérature en philosophie de l'art. Dans une première section nous synthétisons les éléments de cette analyse sur la danse, dans la seconde nous présentons brièvement le cadre théorique à partir duquel nous avons conduit les analyses micro-didactiques, objet de la troisième section.

\subsection{Les œuvres et le rapport aux œuvres en danse contemporaine}

7 «À partir de quand parle-t-on de danse? Il s'agit de choses qui se réfèrent à la conscience, à la perception de son propre corps (...) et à la façon d'agir sur les choses. 
On n'a pas besoin pour cela de la forme esthétique traditionnelle. Ça peut être différent et être toujours de la danse » (Pina Bausch citée par Félix, 2011).

8 Cette citation de la célèbre chorégraphe contemporaine pose d'emblée l'œuvre en danse contemporaine comme ne relevant pas d'une "œuvre académique » au sens commun du terme comme ont pu l'être les livrets des ballets classiques, ce qui réinterroge le statut de l'œuvre en danse. Une revue de questions, empruntant aux philosophes de l'art (Beauquel \& Pouivet, 2010 ; Goodman, 1988) permet d'ébaucher une problématique du rapport aux œuvres permettant de questionner cette dimension dans le champ scolaire. Les paragraphes suivants tentent de clarifier d'un point de vue épistémologique, ce qui «fait danse artistique » et ce qui « fait œuvre ».

Tout comme Goodman (1988) prend le parti de décrire sous quelles conditions il y a art, et non de définir ce qu'est l'art, nous tentons, en nous appuyant sur une revue de littérature en philosophie de l'art et de la danse, d'identifier quand y-a-t-il danse artistique. La philosophie de l'art définit la danse comme la mise en jeu et le déplacement (ou non) de tout ou partie du corps selon des métamorphoses perpétuelles. La danse dans ce sens ne peut être réduite à une activité de production de formes comme le définissent les programmes EPS (MEN, 1996 et 2008a). Pour qu'il y ait danse, le geste doit être juste et « auto-affecter » le danseur (Beauquel \& Pouivet, 2010 ; Bernard, 2001 ; Félix, 2011 ; Guisgand, 2010 ; Kintzler \& Boissière, 2006). Pouivet (2010) la considère comme une activité humaine intentionnelle car ajoute-t-il «les lapins ne dansent pas». Cet acte intentionnel a pour but de communiquer avec autrui selon différentes gestualités possibles, car « le geste artistique de danser est un processus de remaniement structurel, contextuel et sémantique grâce auxquels il acquiert une signifiance autre" (Félix, 2011, p. 43). Ces considérations soulignent que la danse artistique combine une dimension poḯtique, c'est-à-dire relative aux procédures de production artistique, et une dimension aesthésique, c'est-à-dire liée à sa réception en terme de sensibilité esthétique (Bernard, 2001; Michaud, 1999). Les dimensions poḯtique et aesthésique, ici reprises par ces deux auteurs pour les œuvres chorégraphiques, caractérisent selon la philosophie contemporaine toute œuvre d'art.

La poursuite de l'analyse épistémologique nous amène à identifier ce qui, en danse, fait œuvre. Selon la problématique des beaux-arts, pour qu'il y ait art, il doit y avoir production d'œuvres. Ainsi, la danse artistique est « un art du corps engagé dans la production d'objets inouïs proposés publiquement " (Kintzler, 2010). Par ailleurs une œuvre - ici une œuvre chorégraphique - a pour caractéristique d'être toujours adressée! Le spectateur attribue une signifiance à ce qu'il reçoit fondée sur une empathie kinesthésique de nature esthétique (Félix, 2011 ; Michaud, 1999 ; Pouillaude, 2009). La dimension communicative est ainsi le troisième élément indispensable pour prétendre au statut de danse artistique. Mais alors, sous quelles conditions une œuvre peut-elle prétendre au statut d'œuvre d'art?

11 À la suite de Pouivet (2010) on considérera les œuvres d'art comme «des artéfacts culturels dont le fonctionnement spécifique esthétique détermine la nature spécifique au sein des œuvres » (p. 61). Mais, au-delà de ce critère de fonctionnement esthétique, une œuvre d'art doit aussi être reconnue en tant que telle par une communauté autorisée: critiques, auteurs, spécialistes (Danto, 1989). Du point de vue de la philosophie de l'art, les auteurs considèrent enfin, que si en effet les œuvres ont des intentions, on n'accède cependant pas aux intentions d'une œuvre par simple frayage 
mais par apprentissage (Pouivet, 2000, 2010) ce qui nécessite des institutions. Nous ajouterons, en suivant en cela Chevallard (1997), des institutions didactiques !

Ces perspectives soutiennent d'une certaine manière les préconisations actuelles en matière de formation artistique dans le système scolaire, qu'il s'agisse de la danse en EPS, comme nous l'évoquions en introduction de cet article (MEN, 2008a), mais aussi des nouveaux objets curriculaires pluridisciplinaires introduits dans les programmes du collège sous l'appellation « Histoire des arts » (MEN, 2008b). Pour autant, soulignons que ces deux textes échouent à définir clairement les attentes explicites d'un tel projet éducatif. Or, cette dimension de la formation artistique est hautement symbolique car elle renvoie à des choix démocratiques d'accès à la culture pour l'ensemble des élèves qui ne s'actualisent pas facilement dans les pratiques (Mili \& Rickenmann, 2004, 2005). Dans une perspective de transposition didactique, ces auteurs soulignent que la construction sociale de l'expérience esthétique en milieu scolaire, relativement à l'enseignement des disciplines artistiques, doit "permettre aux élèves de construire leur propre rapport à ce type d'objet [les œuvres culturelles] à partir d'une accentuation de la distance à: ils doivent produire une version et pas une copie» (Rickenmann, Mili \& Lagier, 2009, p. 139). Ainsi, la construction de rapports expérientiels aux œuvres d'art (plastique, musicale, théâtrale, chorégraphique, etc.) doit être considérée depuis la perspective pragmatique des usages sociaux qui se construisent à l'École au sein d'activités collectives en lien avec la production des élèves dans ces domaines. Cette problématique est centrale pour la danse en EPS dont le curriculum, contrairement à l'Histoire des arts, spécifie que cette discipline n'est pas le lieu d'étude des œuvres chorégraphiques de répertoire (Tribalat, 1995). Les œuvres qui y sont abordées en référence aux savoirs et pratiques culturelles de la danse sont celles produites par l'enseignant (notamment les «propositions dansées » transmises aux élèves) ou par les élèves (notamment les compositions chorégraphiques collectives qui doivent être performées en fin de séance ou de cycle).

13 Ces développements nous amènent à conclure qu'en danse en EPS, si les productions chorégraphiques des élèves peuvent être considérées comme des œuvres au sens de Meyerson (1948) et peuvent avoir un fonctionnement esthétique, elles ne peuvent pour autant prétendre au statut «d'œuvre d'art ». Il en va de même pour les propositions dansées des professeurs. Pour autant lors du curriculum en actes, ces deux types de production sont susceptibles d'initier chez les élèves des transformations de leur rapport aux œuvres en danse, que l'analyse épistémologique nous amène à définir comme tout ce que les élèves peuvent dire, faire ou savoir à propos des œuvres chorégraphiques auxquelles ils sont confrontés que ce soit en tant que danseur, chorégraphe ou spectateur. Ayant brossé à grands trait la réflexion permettant de conduire l'étude didactique, envisageons maintenant l'inscription théorique de la recherche.

\subsection{Une analyse ascendante de la transposition didactique : inscription théorique de la recherche}

14 Étudier la production du curriculum par les acteurs en situation est au cœur de la démarche. Elle consiste, depuis les recherches pionnières de Marsenach et Mérand (1987) à considérer que les contenus d'enseignement émergent en situation lors des interactions du professeur avec les élèves. Cette perspective, développée en didactique 
comparée, considère que «l'investigation des phénomènes transpositifs [ne peut être] ni (que) dans les savoirs, ni (que) dans les sujets - enseignants et apprenants - mais dans leur action conjointe (...) pour considérer ce travail de coproduction de connaissances à la lumière des pratiques culturelles qui le légitiment " (SchubauerLeoni \& Leutenegger, 2005, p. 408). Il ressort de cette assertion que l'étude des phénomènes transpositifs doit être menée au carrefour d'analyses épistémologiques et interactionnelles (Amade-Escot, 2007). Si les actions du professeur et des élèves sont en effet contingentes et enchâssées, elles sont aussi déterminées par le choix des objets de savoir mis à l'étude et des pratiques de référence qui les légitiment (Martinand, 1986). Dans le domaine de l'art chorégraphique et au regard de l'analyse précédente, il nous semble cependant davantage pertinent de parler "d'œuvres de référence » si l'on souhaite prendre au sérieux les ambitions curriculaires annoncées.

15 À l'échelle micro-didactique des interactions en classe, pour rendre compte de la manière dont dans l'action conjointe avec ses élèves, l'enseignant observé rend possible une entrée dans un rapport poïétique et aesthésique à l'œuvre chorégraphique (Bernard, 2001), nous avons mobilisé les descripteurs de l'action conjointe en didactique (Sensevy, 2007). D'un point de vue formel, ce cadre d'analyse est structuré autour de quelques positionnements théoriques auxquels sont associés des descripteurs permettant d'analyser les corpus. Centré sur une approche pragmatique et située des pratiques didactiques, il a pour visée de rendre compte des configurations et des trajectoires des savoirs enseignés en relation avec l'agentivité déployée par les acteurs. Trois concepts relèvent du noyau dur théorique: celui de transposition didactique, celui de contrat didactique, celui de milieu didactique. La portée de ces trois concepts est de mettre au jour les dynamiques évolutives du curriculum en actes. Par exemple, certains éléments du milieu et du contrat didactiques initialement fixés par le professeur évoluent inévitablement lors des interactions avec les élèves, mais aussi entre élèves. Pour rendre compte de ces évolutions, plusieurs descripteurs considérés comme des outils analytiques sont convoqués: ceux qui décrivent l'action du professeur, ceux qui rendent compte - à partir des dimensions langagières (verbales et non-verbales) - de la manière dont sont co-construits les savoirs dans l'action conjointe. Pour ce qui est des descripteurs de l'action du professeur, Sensevy (2007) distingue quatre modalités : définir, dévoluer, réguler, institutionnaliser. L'action de définition permet d'indiquer aux élèves ce qui est attendu d'eux. L'action de dévolution consiste à amener les élèves à prendre des responsabilités dans la production du savoir visé. L'action de régulation, étroitement liée aux manières de faire des élèves, a pour fonction essentielle de gérer au fil des interactions les incertitudes inhérentes aux situations d'apprentissage. Enfin, l'action d'institutionnalisation désigne le processus par lequel le professeur assure le passage de ce qui est vécu individuellement, et de ce fait très singulier, à un savoir qui sera considéré comme légitime dans la classe. Ce quadruplet d'actions ne doit cependant pas être considéré de manière isolée. Il est associé avec un deuxième type de descripteurs rendant compte de la genèse des savoirs dans la classe : la mésogenèse rend compte de l'évolution du système des objets coconstruits par l'enseignant et les élèves lors des interactions ; la topogenèse caractérise le partage des responsabilités (ou topos) prises en charge par les acteurs relativement aux objets de savoir ; la chronogenèse traite du mode d'avancement des objets de savoir au cours du temps didactique. "A tout état de la mésogenèse, correspond un état du partage des responsabilités entre le professeur et les différents élèves (topogenèse) en lien avec l'avancée ou la stagnation du temps didactique (chronogenèse) eux-mêmes 
dépendants des cadres épistémiques (relatifs au savoir en jeu) et interactionnel (forme des échanges) qui en structure la dynamique " (Amade-Escot \& Venturini, 2009, p. 29). Ayant brossé à grands traits le cadre théorique, nous précisons ci-après les principes méthodologiques mis en œuvre pour mener l'enquête.

\subsection{Dispositif méthodologique}

Pour documenter la manière dont, dans l'action conjointe avec ses élèves, l'enseignant observé met à l'étude les injonctions du programme sur la « construction d'un rapport culturel aux œuvres » et l'évolution des savoirs y afférant au fil des six années et des quatre cycles de danse de l'étude longitudinale, nous avons opté pour une approche multidimensionnelle de recueil des données selon trois échelles temporelles et trois granularités d'objets. Une première échelle de type macro-didactique examine la structuration des quatre cycles et des sept ou huit séances qui le composent au fil du temps. Elle a pour but de repérer selon un grain très grossier les grandes lignes des savoirs mis à l'étude et leurs liens avec les préconisations curriculaires, notamment celles relatives à la formation des trois rôles de danseur, chorégraphe et spectateur. Cette approche macroscopique cherche aussi à identifier quelles sont les tâches récurrentes mises en œuvre dans les séances et dans les cycles qui pourraient être candidates (tertium comparationis) à l'analyse micro-didactique, seule à même de rendre compte de la manière dont se co-construit in situ le rapport aux œuvres en danse. La figure ci-après rend compte du dispositif méthodologique.

Figure 1 : Modalité de l'enquête longitudinale selon trois échelles temporelles et trois granularités d'analyse

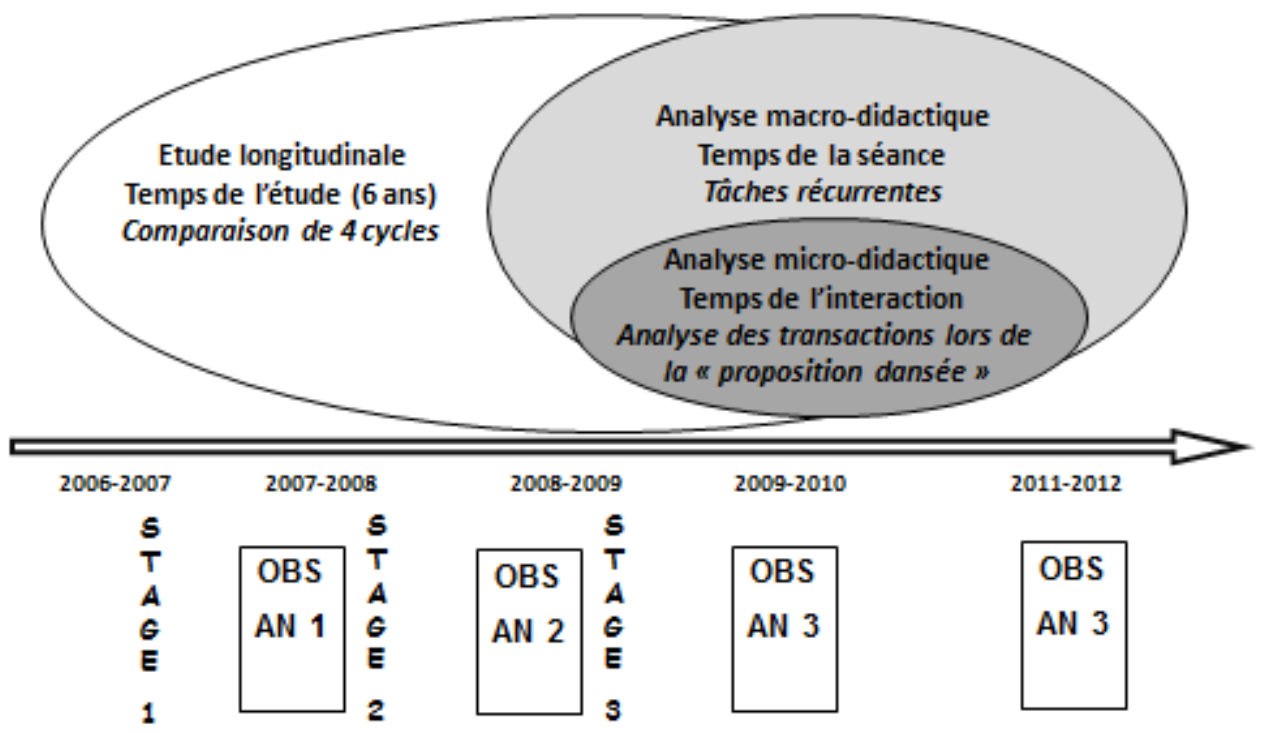

Pour mener l'analyse micro-didactique, nous nous sommes appuyées sur la démarche méthodologique, encore appelée "clinique des systèmes didactiques ", développée par Leutenegger (2009). Pour chaque cycle, nous avons recueilli des données d'entretiens et 
des données d'observation portant sur plusieurs séances consécutives situées en milieu de cycle, au sein desquelles la tâche récurrente de transmission de la "proposition dansée » est proposée aux élèves. Selon les usages méthodologiques de la recherche en didactique nous avons procédé à une analyse a priori de cette tâche. Cette analyse, indépendante de la contingence, a pour fonction d'identifier les enjeux de savoirs cristallisés dans la tâche ainsi que certaines difficultés potentielles que pourraient y rencontrer les élèves.

Pour toutes les séances et les cycles, les données d'observation relatives à la "transmission d'une proposition dansée » (enregistrement vidéo et audio) ont été consignées dans des formats permettant de mettre en relation la description des actions et la transcription des échanges. Nous analysons à partir de l'ensemble des traces collectées le contenu de la proposition dansée et la manière dont Pierre la conduit avec ses élèves. Nous étudions plus particulièrement à l'échelle microdidactique les interactions verbales et non verbales entre ce professeur et les élèves. Le corpus principal des données étudiées à cette échelle temporelle est celui de l'action conjointe en classe. Elle fait l'objet d'une description épaisse (Mary, 1998) prenant en compte (du plus large au plus détaillé) des indicateurs relatifs à l'organisation spatiale et sociale des acteurs, des indicateurs relatifs à l'évolution des contrats et des milieux didactiques (aspects matériels et symboliques), enfin des indicateurs langagiers et posturo-gestuels relatifs au professeur et aux élèves en train de danser de façon à pouvoir caractériser les savoirs enseignés, les objets culturels de référence et le rapport à la danse artistique faisant l'objet de ces transactions. Ces analyses qualitatives, croisées avec les données d'entretien, font émerger un ensemble de traits récurrents dont nous rendons compte dans la section qui suit.

\section{Le rapport à l'œuvre dans la transmission de la proposition dansée : analyse de l'action conjointe professeur - élèves}

19 Dans cette section nous présentons deux extraits situés à deux moments significatifs de l'enquête longitudinale mettant en évidence les manières dont, dans l'action conjointe, certains savoirs liés à la tâche " proposition dansée » sont mis à l'étude. Nous débutons cette présentation par l'analyse a priori de la tâche, puis nous rendons compte de l'évolution de sa mise en œuvre par Pierre.

\subsection{Analyse a priori de la tâche : transmission de la proposition dansée}

Cette tâche, mise en place lors de toutes les séances des cycles de Pierre à l'exception de celles consacrées à l'évaluation de fin de cycle, doit être considérée comme prototypique (Harbonnier-Topin, 2012) de la manière dont les savoirs du danseur sont mis à l'étude en EPS. Il s'agit selon les propres termes de l'enseignant d'une tâche visant à " apprendre un module » (entretien post-séance). Il s'agit d'une tâche générique qui, au-delà des visées d'acquisition de différentes formes de mouvement dansé et d'un vocabulaire y afférant, relève aussi de ce qui peut être considéré comme la mise à l'étude d'une œuvre au sens de Meyerson (1948), ici celle produite par le professeur 
pour être transmise. Pour enseigner une proposition dansée, Pierre, tout au long des cycles observés, présente aux élèves un milieu constitué de monstrations réitérées d'un court module de danse (entre 1 et 2 minutes), le plus souvent accompagné verbalement. La "proposition dansée » du professeur, notamment son degré de difficulté technique, ses qualités d'amplitude, d'intensité, de rythmicité, de présence par le regard, définissent le milieu didactique initial à partir duquel se déploie l'action conjointe. Au fil des transactions, les monstrations successives et les énoncés contribuent à le préciser. Cependant, comme le soulignent Harbonnier-Topin (2012) et Faure (2000), le niveau de performation des élèves dans l'apprentissage d'une proposition dansée est aussi conditionné par leurs capacités sensorielles, leur vécu antérieur, leur motivation, le sens attribué à la proposition dansée, la familiarité avec les gestes à reproduire et leur complexité. Dans le «contrat d'imitation» (Sensevy, 2011) proposé par Pierre, plusieurs types de savoirs sont en jeu pour s'approprier la proposition dansée :

- Percevoir les signes pertinents dans la monstration du professeur et les transformer en puissance d'agir.

- Enchaîner les éléments sans les juxtaposer «en une métamorphose sans fin " (Bernard, 2001) pour donner à voir des moments de danse.

Plusieurs difficultés sont identifiables. Une première difficulté pour les élèves réside dans l'instantanéité et la pluralité des actions du professeur et des différentes parties de son corps, rendant le milieu particulièrement dense. Une deuxième difficulté est d'interpréter les signes corporels et discursifs dont fait ostentation le professeur lorsqu'il transmet la proposition dansée afin de leur donner du sens pour se les approprier. Notons que la linéarité du langage et sa diachronie ne peuvent rendre compte du syncrétisme et du synchronisme du mouvement dansé. Nous estimons également "qu'entendre dire " un mouvement dansé constitue une difficulté majeure pour celui qui l'entend pour la première fois et qui n'en a pas l'usage. Dire un mouvement dansé, relève d'un jeu de langage au sens de Michaud (1999) que les élèves de sixième ne possèdent pas dans ce premier cycle de danse et qui nécessite un apprentissage. Une troisième difficulté réside, pour les élèves, dans la possibilité (ou non) d'auto-évaluation de leur performation grâce uniquement aux actions (verbales et non verbales) du professeur, d'autant que le lieu où se déroulent les séances est une salle sans miroir (contrairement aux salles de danse) et donc en absence de rétroactions visuelles. Une quatrième difficulté provient enfin du " contrat d'imitation " qui force un rapport effectif au milieu pour l'élève tentant d'agir adéquatement. Si le risque physique est peu probable, le risque symbolique est toujours présent: celui d'imiter maladroitement la proposition et d'être moqué par ses pairs. Certains élèves, face à ce risque, peuvent sur-jouer ou faire les clowns ou au contraire n'osent pas danser. 
Figure 2 : Synthèse de l'analyse a priori de la tâche « enseigner-apprendre une proposition dansée

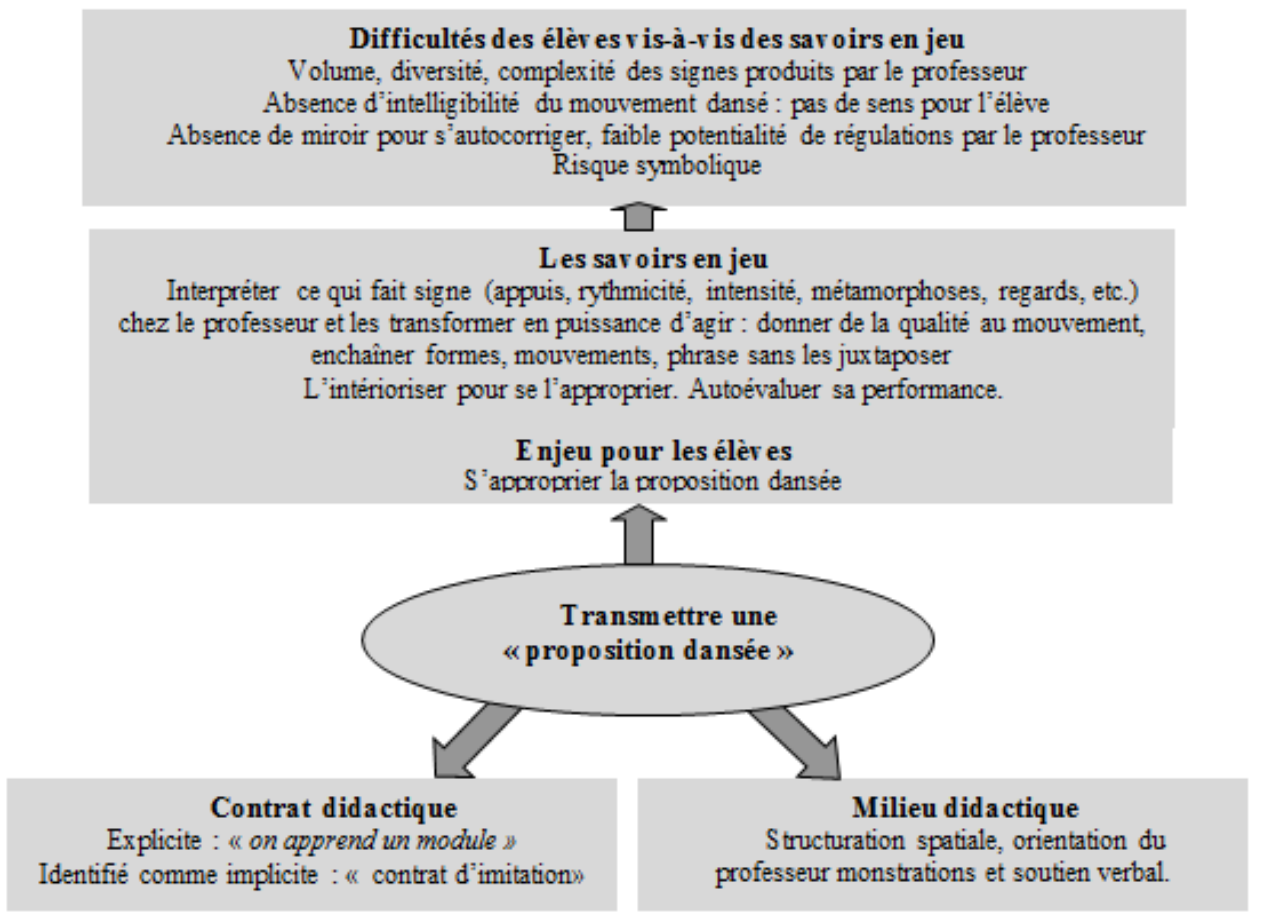

\section{2. Évolution des savoirs mis à l'étude}

Suite à un premier stage de formation continue suivi en 2007 (cf. figure 1), Pierre programme un premier cycle de danse avec une classe de sixième. Il organise son intervention à partir des éléments travaillés lors du stage. Il structure les séances autour de trois grandes tâches récurrentes sur tout le cycle: une tâche visant à "apprendre individuellement un module dansé", une tâche où il s'agit de "composer collectivement une courte chorégraphie " à partir de quelques consignes, une tâche enfin, de performation de la "chorégraphie collective devant le reste de la classe " placée en position de spectateurs (entretien avec Pierre). Ces trois tâches reprennent les trois enjeux des programmes visant la formation du danseur, du chorégraphe et du spectateur. Nous nous intéressons ci-après à la manière dont le curriculum en actes est produit dans l'action conjointe professeur-élèves lors de la transmission de la proposition dansée. Nous prenons appui pour ce faire sur deux extraits jugés caractéristiques de son évolution tout au long des différents cycles et séances observés. Nous concluons la présentation par un tableau de condensation des résultats que nous commentons dans la section discussion.

\subsubsection{La transmission de la proposition dansée selon un contrat didactique de " monstration-reproduction"}

Nous retenons un extrait tiré de la séance 2 du cycle mis en place en 2007. Placé face aux élèves dans une position dite "en miroir", Pierre accompagne verbalement la monstration de sa proposition dansée. Composée de cinq phrases, sans difficulté technique, elle est davantage une juxtaposition d'actions quotidiennes que de «formes gestuelles" (comme y incitent les programmes EPS). Si la position en miroir a 
l'avantage pour l'enseignant de pouvoir observer les réalisations des élèves et de réguler leur prestation par des rétroactions, pour les danseurs elle complexifie la prise d'informations et pose un problème de référentiel spatial. Lorsque Pierre dit «bras droit » et montre le geste avec son bras droit, plusieurs élèves lèvent leur bras gauche pour rester en miroir avec le professeur. Le milieu initial proposé est donc particulièrement contre-intuitif pour les élèves qui doivent ajuster leur action aux signes produits par Pierre. Ce milieu laisse en effet totalement à leur charge le travail d'inversion de latéralité.

L'analyse des registres lexicaux mobilisés lors de ce premier cycle par Pierre pour accompagner sa monstration ou réguler les actions des élèves indique qu'il utilise majoritairement et de façon pertinente des verbes d'actions : « ... on se relève...on passe au sol » (verbatim de séance). Les élèves suivent au plus près les indications verbales de Pierre. Selon Harbonnier-Topin (2012) la fonctionnalité des verbes d'action est de proposer d'emblée une « gestalt » à propos de la coordination globale de la proposition dansée ce qui, selon cette auteure, favoriserait une auto-organisation du corps permettant à l'élève d'agir sans passer par une analyse cognitive spatiale et temporelle coûteuse. Certains verbes, comme "descendre", condensent en effet une double indication: sur l'action à entreprendre (plier les genoux) et sur la direction (vers le bas). Le registre lexical d'accompagnement de Pierre, pour sa part, associe aux verbes d'action des mots désignant les parties du corps à mobiliser: "On va rapprocher nos mains, on les frotte» (verbatim de séance). La faible technicité des registres lexicaux utilisés par cet enseignant non spécialiste contribue à produire des descriptions selon une sémantique familière, peu technique, peu précise : "nos mains bougent pas, c'est le corps qui bouge " (verbatim de séance). Cette formulation témoigne de la difficulté de Pierre à situer de façon précise les parties du corps qui initient le mouvement. Lors des observations effectuées la première année, nous avons observé seulement deux recours à une analogie porteuse de sens: "on va faire comme si on s'appuyait sur l'air». Or, Harbonnier-Topin (2012) souligne toute la pertinence des métaphores qui permettent de substituer à une description biomécanique, longue et indigeste, une comparaison imaginaire. Par cette action de définition, Pierre signifie que l'air pourrait servir de support fixe et invisible pour l'appui des mains. Cette condensation de sens (Tribalat, 1995) permet à Pierre d'accompagner sa monstration sans interrompre son mouvement tout en ajoutant une dimension poétique au milieu didactique en cours d'évolution. Le registre lexical relatif à la description dynamique du mouvement (regard, rythmicité, intensité, appuis, états de corps) n'est en revanche pratiquement pas mobilisé dans le discours d'accompagnement de la proposition dansée. Par exemple, Pierre utilise deux fois le terme «doucement » pour tenter de qualifier la vitesse d'exécution du rapproché de bras et du relevé. Mais ce terme reste porteur d'ambigüité pour les élèves, car «doucement» renvoie, selon les paramètres de Laban (1948/1994), davantage à l'intensité consentie au geste qu'à sa vitesse. Par ailleurs, sur le registre gestuel lorsque Pierre accélère la rotation du bras dans la phrase 5 , il ne l'indique pas aux élèves, tout comme dans la phrase 1 lorsqu'il laisse tomber ses bras en les abandonnant à la gravité, alors que l'intention didactique de Pierre est de jouer sur des changements d'amplitude, de vitesse: "dans la logique ce mouvement est lent et j'aurais aimé que les grands mouvements de bras et le pas chassé deviennent rapides " (entretien post-séance). Il laisse ainsi aux élèves la charge de percevoir ces signes dans sa monstration. Ces derniers, démunis pour le faire n'ont d'autres possibles, pour répondre aux attentes de leur professeur, que de (re)produire des mouvements incorrects et approximatifs, 
notamment pour le pas chassé, pour lequel la plupart d'entre eux effectuent un saut latéral. Une première hypothèse interprétative pourrait être que Pierre juge sa monstration suffisante pour que les élèves s'approprient le mouvement. Une deuxième hypothèse serait que Pierre ne possède pas les savoirs pour transmettre ce qui en danse est appelé : "qualité du mouvement » ou encore "éléments de profondeur " (Vellet, 2006). La dernière hypothèse, plus probable, est que ce professeur n'est pas encore en mesure, à l'issue d'un premier stage de formation, de mobiliser les différents registres de descriptions possibles pour accompagner une monstration. Or, l'élaboration d'un milieu didactique adéquat à la production d'un mouvement dansé réside en partie dans la diversité des registres sémiotiques utilisés par le professeur. Nous considérons que le nombre d'occurrences de verbes d'action, le peu d'éléments sur la qualité à donner au mouvement sont des indicateurs soutenant l'interprétation selon laquelle Pierre, lors de ce premier cycle, valorise dans l'action conjointe avec ses élèves, une description cinématique du mouvement (selon les procédés en usage dans l'enseignement de l'EPS et du sport en général). De ce type d'accompagnement verbal, peu précis, résulte un contrat implicite de reproduction mimétique d'un modèle gestuel (Marsenach \& Mérand, 1987) faiblement porteur des éléments de profondeur du mouvement et des dimensions communicatives et aesthésiques qui caractérisent une performation dansée. Ce faisant, le contrat didactique ne permet pas aux élèves d'établir un rapport de « distance à » l'œuvre performée pour reprendre les termes de Rickenmann, Mili \& Lagier (2009).

\subsection{2. Évolution dans la transmission de la proposition dansée : d'une reproduction formelle à une « monstration-appropriation »}

Dans cet extrait de séance du cycle observé en 2009 (cf. figure 1), la proposition dansée de Pierre présente elle aussi peu de difficultés techniques. Elle est composée d'une juxtaposition de verbes d'actions quotidiennes: lancer, jeter, regarder, attraper... Cependant, par le jeu avec la pesanteur, par les modulations d'intensité, de rythmicité, par le travail sur le placement du regard contribuant à donner de la qualité et de la présence au mouvement, Pierre produit dans sa monstration des moments de danse absents lors de la première année d'observation. Si nous notons encore quelques maladresses dans ses mouvements, soulignons qu'en adoptant une position dos aux élèves, prototypique de la transmission d'une proposition dansée selon HarbonnierTopin (2012), Pierre élimine les problèmes de latéralisation, rencontrés par les élèves lors du premier cycle.

L'évolution la plus prégnante concerne les dimensions mésogénétiques de l'accompagnement verbal de la monstration. Pierre débute sa proposition dansée en disant: " on attrape quelque chose... et ce quelque que chose vous savez ce que c'est ?... c'est une balle». Il s'agit d'une "métaphore filée » (Commandé, 2011) à propos d'une balle imaginaire qui est utilisée tout au long de ce cycle. Le processus de symbolisation sousjacent à l'usage de métaphores a pour but, selon cette auteure, de transporter les élèves dans un monde fictif et de donner du sens aux mouvements. Nous notons dans les énoncés de Pierre que les occurrences de verbes d'action augmentent de façon significative (55) de même que ceux relatifs à la description cinématique du mouvement (25) : «On ferme, on s'accroupit, on recule le pied droit... » (verbatim de séance). S'ajoutent à cet ensemble d'énoncés descriptifs, des verbes que nous avons appelés métaphoriques parce qu'ils contribuent à maintenir vive la métaphore de la balle 
imaginaire : «lancer, attraper, bloquer... » (30 occurrences dans cet extrait). L'objet de ces verbes métaphoriques n'est pas, comme pour les précédents, de désigner la partie du corps à mobiliser (i.e. " on ferme la main »). Ils indiquent l'action à effectuer en référence à la métaphore filée de la balle imaginaire : « on va la lancer et la récupérer... je la bloque.... Je la ramène... " (verbatim de séance). D'un point de vue mésogénétique ces verbes transposent le sens initial du mouvement ("lancer le bras») au-delà, dans le champ sémantique de la balle. Nos observations pointent que certains élèves lancent la balle fictive loin en avant et l'accompagnent du regard, comme pour évaluer la distance de leur lancer; d'autres la lancent très haut, sans la regarder, et se protègent la tête lors de sa chute imaginaire. Ces observables documentent d'une part, la qualité du mouvement performé par les élèves danseurs et d'autre part, les significations construites dans l'action conjointe. Ils confortent le qualificatif de métaphorique que nous attribuons au soutien verbal du professeur. La première fonction des verbes métaphoriques utilisés par Pierre est d'indiquer l'énergie à consentir pour la réalisation de l'action et sa rythmicité. Les verbes lancer, attraper, bloquer sont ainsi porteurs d'une composante vitesse « rapide » et d'une composante énergie «forte » en début d'action. Ils sont également un moyen délibéré pour Pierre de concentrer l'information : "et du coup ça suit derrière il n'y a pas besoin de dire lent vite et machin, ils pigent... » (entretien post- séance). Leur deuxième fonction pourrait être de fournir aux élèves une intention pour leur action, leur permettant de passer d'une simple production de formes, ce que Paillard (1971) nomme "morphocinèses", à une production de sens que Serre (1984) définit comme des "sémiocinèses ", c'est-à-dire des formes motrices présidant aux relations entre l'homme et son environnement social à des fins de communication symbolique.

"Lancer la balle, la bloquer " (verbatim) nous semble être dans cette séance le moyen retenu par Pierre pour réactiver une expérience connue par tous, expérience constitutive du milieu didactique proposé aux élèves. Ces ingrédients du milieu portent l'intentionnalité didactique visant à dévoluer aux élèves la responsabilité de la qualité de leur mouvement. Le ressenti du mouvement touche l'élève par inférence et celui-ci passe d'une production de formes extérieures à un mouvement éprouvé. Pierre crée ici les conditions pour rendre possible un changement de rapport à la danse, ce que Guisgand (2010) désigne par "état de corps ", à savoir l'ensemble des tensions et intentions qui s'accumulent intérieurement et vibrent extérieurement.

La mésogenèse s'enrichit aussi par le truchement de prosodies: «on va faire baaaasculeeer la balle", d'interjections: "et hop ", de glossolalies: "ouaaahhhhh" (verbatim de séance, en gras les accentuations toniques). Pierre renforce ainsi les sémioses relatives à la proposition dansée. Il met l'accent sur les traits pertinents qu'il s'agit pour les élèves, non de reproduire, mais d'actualiser dans leur propre mouvement dansé. Les signes de la monstration, ici, ne relèvent plus de la cinématique du modèle gestuel à reproduire, mais concernent les intensités et rythmicités du mouvement.

Prosodies, interjections, glossolalies agissent en synergie avec l'accompagnement verbal. Le choix de la métaphore filée à propos de la balle fait milieu. Les élèves peuvent se saisir du jeu dans lequel Pierre joue sa partition. Il projette ainsi un modèle d'identification analogique significatif pour les élèves pour qu'ils se saisissent des intentions de l'action. Pierre avance dans l'action conjointe avec eux dans une sémiose partagée (Sensevy, 2011). Le procédé de la métaphore filée, initié lors de ce cycle, 
s'enrichira tout au long des années observées, des propositions dansées de Pierre et de l'imaginaire des élèves. Le milieu didactique conjointement produit crée ainsi les conditions pour que les élèves dansent et, ce faisant, transforment leur rapport à l'œuvre en rendant possible une prise de «distance» avec les gestes usuels de manipulation de balle. Cette prise de distance (Rickenmann, Mili \& Lagier, 2009) indique un changement de rapport à l'œuvre dansée pouvant être ici compris comme une contribution à leur développement (Meyerson, 1948) en termes de formation artistique.

Rappelons aussi que l'usage de verbes métaphoriques relève d'une pratique professionnelle spécifique à la transmission artistique (Harbonnier-Topin, 2012; Loquet, 2006). Contrairement à la danse classique, les consignes descriptives laissent place, en danse contemporaine, à des consignes imagées permettant de travailler selon un registre culturel de rapport à l'œuvre (Mili \& Rickeman, 2005). Cet usage nouveau, inauguré en 2009, naît de la pratique d'enseignement et la nourrit en retour. Pierre réinvestit, les expériences réalisées lors des stages de formation continue, comme il nous l'a indiqué lors d'un entretien. Nous pointons ainsi une évolution par rapport au premier cycle mis en place. Le contrat de monstration-reproduction se trouve transformé dans l'action conjointe en un contrat de monstration-appropriation sous l'approbation tacite de Pierre qui laisse de grandes marges de liberté aux élèves quant à la réalisation cinématique du mouvement, alors qu'il ne transige pas sur sa qualité (Vellet, 2006). En privilégiant dans ses définitions et régulations, certains éléments de profondeur de la proposition dansée, Pierre permet aux élèves d'accéder aux dimensions communicationnelle et aesthésique caractéristiques d'un mouvement dansé. Cette tendance se confirme au fil des ans comme le tableau ci-après en rend compte.

Tableau 1 : Transmission de la proposition dansée : évolution des savoirs mis à l'étude par Pierre

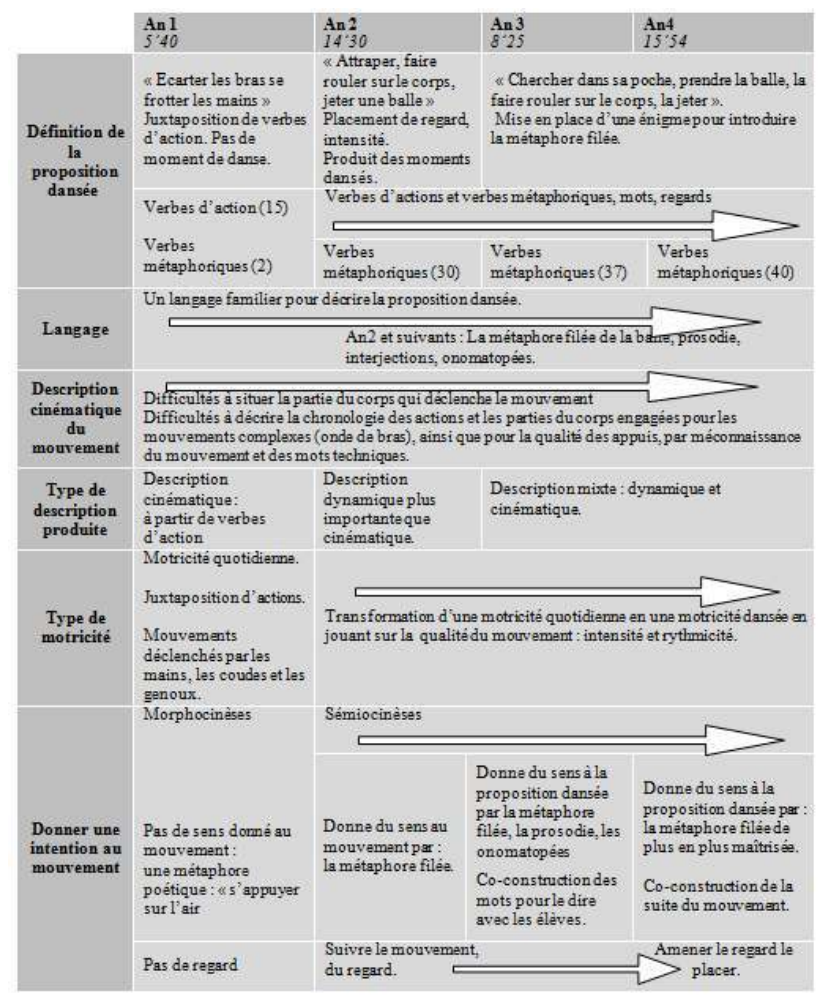


31 Ce tableau synthétise la manière dont Pierre fait évoluer les savoirs mis à l'étude lorsqu'il transmet une proposition dansée. Au fil du temps et des expériences coconstruites dans l'action conjointe avec les élèves apparaissent des inflexions qui, pour certaines, semblent s'installer dès l'an 2. Lorsqu'elles se stabilisent nous l'indiquons par une flèche qui rend compte des permanences alors observées. Nous commentons ces résultats dans la section suivante.

\section{Discussion conclusive}

32 Dans cette discussion nous revenons sur les éléments les plus saillants des évolutions observées, notamment celles qui participent à la construction par les élèves d'un nouveau rapport aux œuvres en danse au collège. Ces éléments et leur mise en perspective sont l'occasion de conclure en quoi l'étude longitudinale permet de mettre au jour les contraintes et l'autonomie du curriculum en actes.

33 Il ressort du tableau précédent que la proposition dansée, dans sa forme gestuelle, reste relativement identique que ce soit en termes de forme des mouvements, d'utilisation de l'espace haut et bas (passage au sol), d'absence de difficultés techniques, ou encore du vocabulaire gestuel mis à l'étude. Toutefois l'analyse de l'action conjointe pointe qu'au fil des ans ce professeur crée des conditions de plus en plus favorables à la production par les élèves de courts « moments dansés » contribuant à transformer leur rapport à la danse et aux œuvres. Ces évolutions, mises en évidence à partir d'une analyse fine des modalités de monstration et des formes langagières de leur accompagnement par Pierre, rendent compte d'évolutions profondes tant au niveau des types de contrats didactiques que des ingrédients des milieux proposés aux élèves. Une première rupture est opérée lorsque Pierre réalise, à l'issue de la première année, les difficultés de latéralisation qu'engendre son orientation face aux élèves les contraignant à se concentrer sur une inversion de la latéralité des mouvements et sur une reproduction mimétique du module, qui va à l'encontre de son intention didactique.

34 Au regard des expériences successives de mise en œuvre de cycles de danse avec des classes de sixième, Pierre - soutenu par la participation à trois stages de formation continue dans lesquels il puise certains matériaux pour l'enseignement (la structure des séances, des documents directement utilisables, des repères concrets pour l'observation de l'activité des élèves) - devient en mesure de produire des milieux didactiques de plus en plus pertinents caractérisés par :

35 la reprise des mêmes propositions dansées, selon une qualité de mouvement de plus en plus fine en termes de rythmicité, d'intensités, de participation du regard, ce que les textes du programme en EPS désignent par vitesse, énergie et présence ;

36 le registre langagier, enrichi par l'usage précis de verbes métaphoriques qui permettent de produire des signes sur les éléments de profondeur du mouvement qu'il souhaite voir produire par ses élèves ;

37 l'usage de métaphores filées pour accompagner ses propositions dansées, qui participent de la création d'un monde fictif que chaque élève peut s'approprier selon son imaginaire, et qui donnent à voir une variété de métamorphoses d'autant plus judicieuses que Pierre régule, au fil des ans, non plus sur la forme du geste, mais sur son sens ; 
enfin, la sensibilité accrue à l'idée que danser, c'est donner à voir les éléments de profondeur du mouvement, les ressentir et en être auto-affecté pour que les collégiens puissent s'engager dans des performations ne scindant pas les dimensions poïétique, aesthésique et communicationnelle du mouvement dansé.

Ces milieux font l'objet, dans l'action conjointe, de transactions dont l'enquête rend compte à partir des différents contrats didactiques qui s'y nouent. L'étude diachronique de l'évolution de ces contrats (cf. tableau 2) met en évidence le passage d'un curriculum centré sur la monstration-reproduction formelle de formes gestuelles juxtaposées, à la mise en œuvre d'un nouveau rapport à la performation en danse. En dévoluant aux élèves un espace de liberté dans l'interprétation de la proposition dansée selon un contrat de monstration-appropriation, Pierre crée les conditions d'émergence des dimensions poḯtique et aesthésique de la danse artistique ayant des effets sur les productions chorégraphiques des élèves que, dans le cadre de ce court article, nous n'avons pu développer.

Tableau 2 : Évolution des contrats didactiques proposés aux élèves dans la transmission de la proposition dansée

\begin{tabular}{|c|c|c|c|c|}
\hline & An 1 & An 2 & An 3 & An 4 \\
\hline $\begin{array}{l}\text { Dynamique } \\
\text { évolutive du } \\
\text { contrat } \\
\text { didactique }\end{array}$ & $\begin{array}{l}\text { Contrat de } \\
\text { reproduction } \\
\text { mimétique de } \\
\text { formes } \\
\text { gestuelles } \\
\text { juxtaposées }\end{array}$ & $\begin{array}{l}\text { Contrat d'ostension- } \\
\text { reproduction tourné } \\
\text { vers la production d'un } \\
\text { mouvement } \\
\text { progressivement plus } \\
\text { dansé }\end{array}$ & $\mid \begin{array}{l}\text { Contrat de } \\
\text { monstration- } \\
\text { appropriation } \\
\text { d'un mouvement } \\
\text { dansé }\end{array}$ & $\begin{array}{l}\text { Contrat de } \\
\text { monstration- } \\
\text { appropriation } \\
\text { malgré une certaine } \\
\text { obsolescence des } \\
\text { milieux didactiques }\end{array}$ \\
\hline
\end{tabular}

Nous considérons que l'évolution de ces formes contractuelles rend possible la mise en place de conditions favorables à l'installation d'un nouveau rapport à la danse chez les élèves. L'analyse et l'interprétation du mouvement dansé tel que co-produit dans l'action conjointe met en effet en évidence un déplacement allant d'une transmission de formes gestuelles à une sémiogenèse collective (Sensevy, 2011). Cette construction du sens dans le collectif de la classe autorise et donne place à une interprétation individuelle de la proposition dansée par chaque élève comme nous l'avons rapporté pour « le lancer de balle».

Pour conclure, cette étude longitudinale sur l'évolution des savoirs enseignés par Pierre montre combien le curriculum en actes résulte de multiples compromis entre contraintes et autonomie. Lors du premier cycle d'enseignement de la danse, en l'an 1, Pierre se saisit des contraintes de surface des programmes: «mémoriser des formes corporelles à reproduire» (MEN, 2008a). Il fait sienne, selon sa propre lecture, cette préconisation d'autant qu'ayant participé à un stage de formation continue il dispose " de billes pour pouvoir se lancer» (entretien préalable à la recherche). Nous avons pu observer que sa pratique d'enseignement consiste alors, via des verbes d'action, à faire mémoriser un enchaînement selon les usages tirés de son expérience de professeur d'EPS, plutôt que de mettre à l'étude des « formes corporelles » (MEN, 2008a). Au fil des ans (mais aussi des stages suivis et des expériences de danse réalisées) ce professeur s'émancipe de cette première lecture du texte officiel en élaborant de nouvelles modalités de transmission des éléments de profondeur du mouvement dansé. En cela, il 
prend en compte de façon originale les préconisations des programmes de sixième. Cette émancipation nous semble traduire une évolution de l'épistémologie pratique de ce professeur (au sens développé par Sensevy, 2007) rendue possible par la reprise, sous des formes sans cesse renouvelées, de l'expérience d'enseignement co-construite avec ses classes de sixième successives.

En choisissant de centrer notre regard sur un moment particulier de la formation du « danseur » dans les séances d'EPS mises en place par Pierre, nous souhaitions montrer comment l'analyse du curriculum en actes impose de prendre en considération les éléments ténus qui sont l'objet des transactions didactiques (vocabulaire gestuel, qualités du mouvement, accompagnement verbal, etc.) au regard des préconisations curriculaires. Il reste que l'analyse longitudinale permet de montrer des évolutions similaires en ce qui concerne la formation des élèves dans les autres rôles sociaux de "chorégraphe " et de "spectateur", tout en pointant l'extrême intrication de ces enjeux de savoirs dès lors que l'on souhaite, comme le préconisent les textes, développer la formation artistique des élèves et la construction par ses derniers d'un «rapport culturel aux œuvres». En s'intéressant au curriculum tel qu'il se fait et évolue au fil des ans, cette étude de cas permet de documenter certaines questions de l'appel à contribution en montrant comment les expériences et les savoirs mobilisés par cet enseignant dans l'action conjointe avec ses élèves influencent la production du curriculum. Plus largement, elle met en exergue les effets de l'expérience dans l'évolution de l'épistémologie pratique du professeur et par voie de conséquence du curriculum effectif.

\section{BIBLIOGRAPHIE}

Amade-Escot, C. (2007). Les savoirs au cœur du didactique. In C. Amade-Escot, (Éd.), Le didactique (pp. 11-30). Paris : Revue EP.S.

Amade-Escot, C., \& Venturini, P. (2009). Le milieu didactique : d'une étude empirique en contexte difficile à une réflexion sur le concept. Éducation et didactique, 3(1), 7-44.

Arguel, M. (1992). Danse. Le corps enjeu. Paris : PUF.

Beauquel J., \& Pouivet R. (2010). Philosophie de la danse. Rennes : PUR.

Bernard, M. (2001). De la création chorégraphique. Paris : Centre national de la danse.

Chevallard, Y. (1997). Questions vives, savoirs moribonds : le problème curriculaire aujourd'hui [CD ROM]. Marseille : Actes du colloque « Défendre et transformer l'école pour tous ».

Commandé, E. (2011). La danse artistique une activité motrice de symbolisation. http:// www.passeursdedanse.fr/pdf/la_danse_artistique_une_activite_motrice_de_symbolisation.pdf Crahay, M., Audigier, F., \& Dolz, J. (2006). Curriculum, enseignement et pilotage. Bruxelles : De Boeck. Danto, A. (1989). La transfiguration du banal : une philosophie de l'art. Paris : Seuil.

Faure, S. (2000). Apprendre par corps. Socio-anthropologie des techniques de danse. Paris : La Dispute. 
Félix, J.-J. (2011). Enseigner l'art de la danse. Bruxelles : De Boeck.

Goodman, N. (1988). Quand y a-t-il art ? In G. Genette (Éd.), Philosophie analytique et esthétique (pp. 199-210). Paris : Klincksieck.

Guisgand, P. (2010). Lire le mouvement dansé. Revue STAPS, 89, 85-89.

Harlé, I. (2009). La fabrique des savoirs scolaires. Paris : La Dispute.

Harbonnier-Topin, N. (2012). Le temps de la transmission en danse : de la construction des savoirs et des pratiques à l'élaboration de traces chorégraphiques. Montréal : UQAM et CRF CNAM.

Kintzler, C. (2010). La danse, art du corps engagé, et la question de l'autonomie. In J. Beauquel \& R. Pouivet (Eds.), Philosophie de la danse (pp. 127-142). Rennes : PUR.

Kintzler, C., \& Boissière, A. (2006). Approche philosophique du geste dansé. Villeneuve d'Asq : Presse universitaire du Septentrion.

Laban, R. (1994). La maîtrise du mouvement. Arles : Actes sud. (Édition originale 1948)

Leutenegger, F. (2009). Le temps d'instruire. Berne : Peter Lang.

Loquet, M. (2006). Analyse des gestes professionnels : illustrations de «l'œil du maquignon » chez une formatrice en expression corporelle. Revue française de pédagogie, 157, 119-130.

Marsenach, J., \& Mérand, R. (1987). L'évaluation formative en EPS dans les collèges. Paris : INRP.

Martinand, J.-L. (1986). Connaître et transformer la matière. Paris-Berne : Peter Lang.

Mary, A. (1998). De l'épaisseur de la description à la profondeur de l'interprétation. À propos de « Thick description ». Enquête, 6, 57-72.

MEN (1996). Programme d'EPS de la classe de sixième des collèges. Bulletin officiel de l'Éducation nationale, 29, 1964-1968.

$\mathrm{ftp}$ ///trf.education.gouv.fr/pub/educnet/musique/neo/04infos/textes/programmes/danse/ bo9001.pdf

MEN (2008a). Programme d'éducation physique et sportive du collège. Bulletin officiel spécial de l'Éducation nationale, 6. http://www.education.gouv.fr/cid22119/mene0817062a.html

MEN (2008b). Organisation de l'enseignement de l'histoire des arts à l'école primaire, au collège et au lycée. Bulletin officiel spécial de l'Éducation nationale, 6.http://www.education.gouv.fr/ cid22078/mene0817383a.html.

Meyerson, I. (1948). Les fonctions psychologiques et les œuvres. Paris : Albin Michel.

Mili, I., \& Rickenmann, R. (2004). La construction des objets culturels dans l'enseignement artistique et musical. In C. Moro \& R. Rickenman (Éds.), Situation éducative et significations (pp. 165-196). Bruxelles : De Boeck.

Mili, I., \& Rickenmann, R. (2005). La réception des œuvres d'art : une nouvelle perspective didactique. Revue suisse des sciences de l'éducation, 27(3), 431-452.

Michaud, Y. (1999). Critères esthétiques et jugement de goût. Nîmes : Jacqueline Chambon.

Montaud, D. (2014). Le rapport aux œuvres dans l'enseignement de la danse au collège. Analyse didactique de l'évolution de l'épistémologie pratique d'un professeur d'EPS. Thèse de doctorat de Sciences de l'Education, non publiée, Université de Toulouse, Toulouse 2 - Le-Mirail, 5 février.

Paillard, J. (1971). Les déterminants moteurs de l'organisation de l'espace. Cahiers de psychologie, 4(4), 261-316. 
Pouillaude, F. (2009). Le désœuvrement chorégraphique. Étude sur la notion d'œuvre en danse. Paris : Vrin.

Pouivet, R. (2000). L'ontologie de l'œuvre d'art. Nîmes : Jacqueline Chambon.

Pouivet, R. (2010). Danse, ontologie et philosophie de l'âme. In J. Beauquel \& R. Pouivet (Éds.), Philosophie de la danse (pp. 33-44). Rennes : PUR.

Rancière, J. (2008). Le spectateur émancipé. Paris : La Fabrique.

Rickenmann, R., Mili, I., \& Lagier, C. (2009). La construction sociale de l'expérience esthétique. Analyse clinique de séquences d'enseignement autour du patrimoine musical et artistique. Les dossiers des Sciences de l'Education, 21, 129-141.

Schubauer-Leoni, M. L., \& Leutenegger, F. (2005). Une relecture des phénomènes transpositifs à la lumière de la didactique comparée. Revue Suisse des sciences de l'éducation, 27(3), 407-429.

Sensevy, G. (2007). Des catégories pour décrire et comprendre l'action didactique. In G. Sensevy \& A. Mercier (Dir.), Agir ensemble, l'action didactique conjointe du professeur et des élèves (pp. 13-49). Rennes: PUR.

Sensevy, G. (2011). Le sens du savoir, Éléments pour une théorie de l'action conjointe en didactique. Bruxelles : De Boeck.

Serre, J.-C. (1984). La danse parmi les autres formes de motricité. La recherche en danse, 3, 135-156.

Tribalat, T. (1995). La danse discipline artistique, Hyper, 192, 21.

Vellet, J. (2006). La transmission matricielle de la danse contemporaine. Revue STAPS, 72, 79-91.

\section{NOTES}

1. Cf. les documents d'accompagnement des textes officiels d'EPS.

\section{RÉSUMÉS}

Sur la base d'une étude de cas longitudinale d'une durée de six ans, cet article examine la manière dont les préconisations curriculaires en EPS relatives à «la formation artistique des élèves » trouvent place dans un enseignement de la danse en classe de sixième. Il s'intéresse à la manière dont un enseignant d'EPS ayant une certaine expérience d'enseignement, mais n'ayant aucune spécialisation en danse, met en œuvre, au fil des ans, les nouveaux objets du programme. A l'échelle micro-didactique, les données exploitées portent sur une phase récurrente dans les cycles observés, celle de la mise à l'étude de la " proposition dansée ». Les résultats montrent que les savoirs et les expériences co-construits dans l'action conjointe évoluent et permettent le passage d'un enseignement de la danse privilégiant un contrat didactique de type « monstrationreproduction » vers un enseignement valorisant des formes contractuelles de "monstrationappropriation ». Cette évolution crée des conditions favorables pour que les élèves puissent transformer leur rapport à l'œuvre en danse dans une sens plus poḯtique, aesthésique et communicationnel. 
danse, éducation physique et sportive, formation artistique, rapport aux œuvres

From the outcomes of a six years longitudinal case study, this paper examines how new dance curriculum standards are implemented in physical education at lower middle school. It looks at the ways the new curriculum is put into practice by an experienced teacher who has no specialization in the field of dance teaching. At the micro-level of didactic interactions, the data concern a recurrent moment of the teaching, when the teacher brings into play a "dance movement". The findings show that content knowledge and experience are co-constructed during didactical joint action and evolve through the years from a teaching based on didactic contracts of "ostension-replication" to didactic contracts of "ostension-construction" in which dancing experiences are enacted through a set of opportunities that allows students to transform their own relationships to dance artwork as more poietic, aesthetic and communicative.

dance, physical education, artistic education, relationship to artwork

\section{AUTEURS}

\section{DOMINIQUE MONTAUD}

Professeure agrégée d'EPS, Docteure en Sciences de l'Éducation, CUFR J-F Champollion, Département STAPS de Rodez, UMR EFTS, MA 122

\section{CHANTAL AMADE-ESCOT}

Professeure en Sciences de l'Éducation, Université Toulouse 2 - Jean Jaurès, ESPE de Toulouse, UMR EFTS, MA 122 